\title{
A review of epileptic seizure detection using machine learning classifiers
}

\author{
Mohammad Khubeb Siddiqui ${ }^{1+} \mathbb{0}$, Ruben Morales-Menendez ${ }^{1 *}$, Xiaodi Huang ${ }^{2+}$ and Nasir Hussain ${ }^{3}$
}

\begin{abstract}
Epilepsy is a serious chronic neurological disorder, can be detected by analyzing the brain signals produced by brain neurons. Neurons are connected to each other in a complex way to communicate with human organs and generate signals. The monitoring of these brain signals is commonly done using Electroencephalogram (EEG) and Electrocorticography (ECOG) media. These signals are complex, noisy, non-linear, non-stationary and produce a high volume of data. Hence, the detection of seizures and discovery of the brain-related knowledge is a challenging task. Machine learning classifiers are able to classify EEG data and detect seizures along with revealing relevant sensible patterns without compromising performance. As such, various researchers have developed number of approaches to seizure detection using machine learning classifiers and statistical features. The main challenges are selecting appropriate classifiers and features. The aim of this paper is to present an overview of the wide varieties of these techniques over the last few years based on the taxonomy of statistical features and machine learning classifiers-'black-box' and 'non-black-box'. The presented state-of-the-art methods and ideas will give a detailed understanding about seizure detection and classification, and research directions in the future.
\end{abstract}

Keywords: Epilepsy, Applications of machine learning on epilepsy, Statistical features, Seizure detection, Seizure localization, Black-box and non-black-box classifiers, EEG signals

\section{Introduction}

The word epilepsy originates from the Latin and Greek word 'epilepsia' which means 'seizure' or 'to seize upon'. It is a serious neurological disorder with unique characteristics, tending of recurrent seizures [1]. The context of epilepsy, found in the Babylonian text on medicine, was written over 3000 years ago [2, 3]. This disease is not limited to human beings, but extends to cover all species of mammals such as dogs, cats and rats. However, the word epilepsy does not give any types of clues about the cause or severity of the seizures; it is unremarkable and uniformly distributed around the world $[1,4]$.

\footnotetext{
*Correspondence: rmm@tec.mx

${ }^{\dagger}$ Mohammad Khubeb Siddiqui and Xiaodi Huang contributed equally to this work

1 School of Engineering and Sciences, Tecnologico de Monterrey, Av. E. Garza Sada 2501, Monterrey, Nuevo Leon, Mexico

Full list of author information is available at the end of the article
}

Several theories about the cause are already available. The main cause is electrical activity disturbance inside a brain $[1,5,6]$, which could be originated by several reasons [7] such as malformations, shortage of oxygen during childbirth, and low sugar level in blood [8,9]. Globally, epilepsy affects approximately 50 million people, with 100 million being affected at least once in their lifetime $[5,10]$. Overall, it accounts for $1 \%$ of the world's burden of diseases, and the prevalence rate is reported at $0.5-1 \%[4,11]$. The main symptom of epilepsy is to experience more than one seizure by a patient. It causes a sudden breakdown or unusual activity in the brain that impulses an involuntary alteration in a patient's behaviour, sensation, and loss of momentary consciousness. Typically, seizures last from seconds to a few minute(s), and can happen at any time without any aura. This leads to serious injuries including fractures, burns, and sometimes death [12].

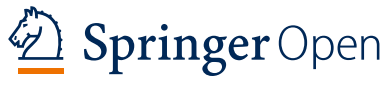

(c) The Author(s) 2020. This article is licensed under a Creative Commons Attribution 4.0 International License, which permits use, sharing adaptation, distribution and reproduction in any medium or format, as long as you give appropriate credit to the original author(s) and the source, provide a link to the Creative Commons licence, and indicate if changes were made. The images or other third party material in this article are included in the article's Creative Commons licence, unless indicated otherwise in a credit line to the material. If material is not included in the article's Creative Commons licence and your intended use is not permitted by statutory regulation or exceeds the permitted use, you will need to obtain permission directly from the copyright holder. To view a copy of this licence, visit http://creativeco mmons.org/licenses/by/4.0/. 


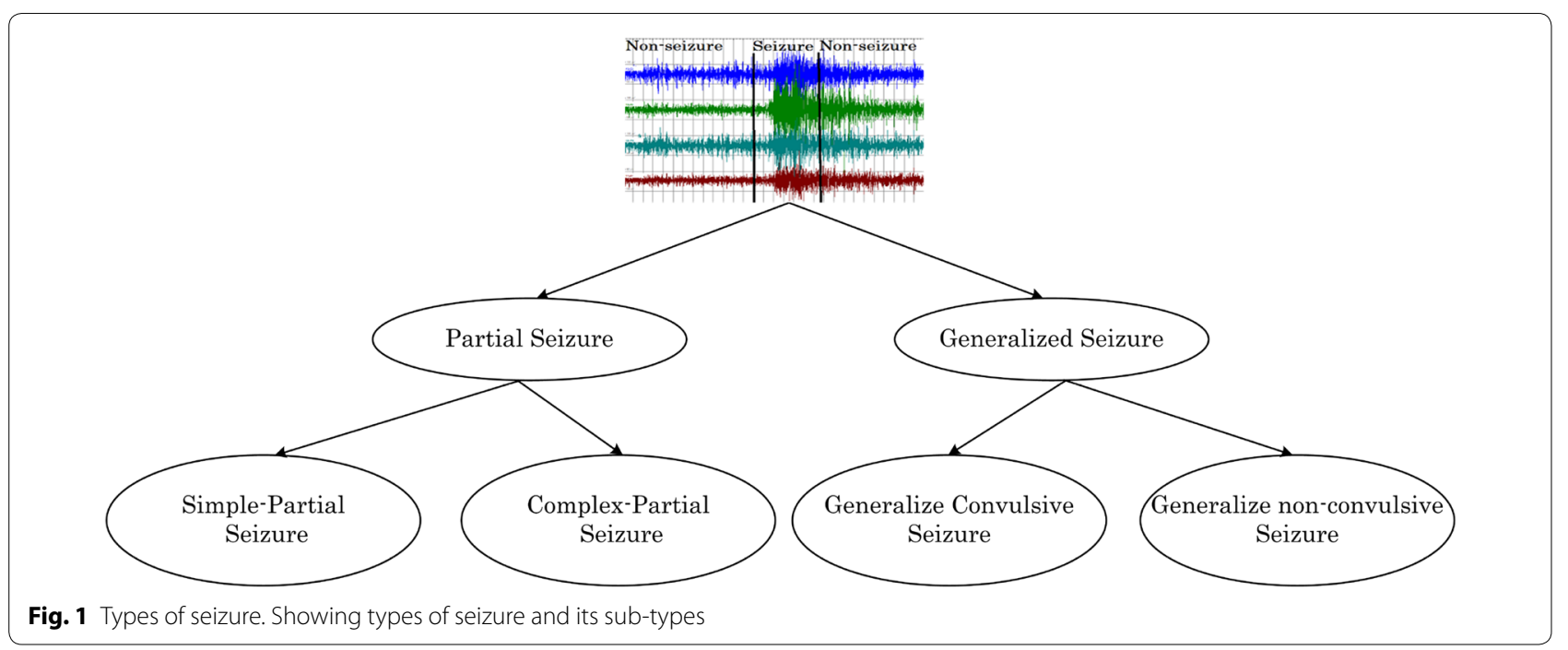

\subsection{Seizure type}

Based on the symptoms, seizures are categorized by neuro-experts into two main categories-partial and generalized $[7,13]$-as shown in Fig. 1. Partial seizure, also called 'focal seizure', causes only a section of the cerebral hemisphere to be affected. There are two types of Partial seizure: simple-partial and complex-partial. In the simple-partial, a patient does not lose consciousness but cannot communicate properly. In the complex-partial, a person gets confused about the surroundings and starts behaving abnormally like chewing and mumbling; this is known as 'focal impaired awareness seizure.' On the contrary, in the generalized seizures, all regions of the brain suffer and entire brain networks get affected quickly [14]. Generalized seizures are of many types, but they are broadly divided into two categories: convulsive and non-convulsive.

\subsection{Main contributions of the paper}

In brief, the contributions of this paper are as follows:

1. We have done the review according to five main dimensions. First, researchers who adopted the EEG, ECoG or both for seizure detection; second, significant features; third, machine learning classifiers; fourth, the performance of the classifier during a seizure, and last, knowledge discovery (e.g., seizure localization).

2. Through study, it has been explored that an ensemble of decision trees (i.e., decision forest-random forest) classifier outperforms other classifiers (ANN, KNN, SVM, single Decision Tree).
3. We also suggest, how decision forest algorithms could be more effective for other knowledge discovery tasks besides seizure detection.

4. This study will help the researchers with their data science backgrounds to identify which statistical and machine learning classifiers are more relevant for further improvement to the existing methods for seizure detection.

5. The study will also help the readers for understanding about the publicly available epilepsy datasets.

6. In the end, we have provided our observations by the current review and suggestions for future research in this area.

The structure of the paper is organized as follows. "Role of data scientists in epileptic seizure detection" section gives the overview of machine learning experts in EEG datasets. The preliminaries requirements are provided in "A framework for seizure detection" section; it presents a general model of seizure detection and explains each step in a subsequent manner. "Publicly available datasets" section provides the details of benchmark datasets with their description. "Seizure detection based on statistical features and machine learning classifiers" section explains the review of literature work done on seizure detection using different machine learning classifiers, with a detailed comparison. "Seizure localization" section reviews the work done in identifying the affected lobes of the brain using machine learning classifiers. In "Problems identified in existing literature" section, we have explored the issues in the previous work and highlighted the gap. Overall, "observation about capable classifiers and statistical features" section reports our observations from the 
review about a suitable classifier and feature. "Research directions in seizure detection" section emphasizes the future directions in this research area, followed by "Conclusion" section on the summary of the paper.

\section{Role of data scientists in epileptic seizure detection}

Applications of machine learning are significantly seen on health and biological data sets for better outcomes $[15,16]$. Researchers/scientists on different areas, specifically, data mining and machine learning, are actively involved in proposing solutions for better seizure detection. Machine learning has been significantly applied to discover sensible and meaningful patterns from different domain datasets $[17,18]$. It plays a significant and potential role in solving the problems of various disciplines like healthcare [17, 19-25]. Applications of machine learning can also be seen on brain datasets for seizure detection, epilepsy lateralization, differentiating seizure sates, and localization [26-29]. This has been done by various machine learning classifiers such as ANN, SVM, decision tree, decision forest, and random forest [26, 28].

Certainly, in the past, numerous reviews have been carried out on seizure detection along with applied features, classifiers, and claimed accuracy [27, 30-33] without focusing on the challenges faced by the data scientists whilst doing research on datasets of neurological disorders. Therefore, this article provides a detailed study of machine learning applications on epileptic seizure detection and other related knowledge discovery tasks. In this review, the collected articles are from well-known journals of their relevant field. These references are either indexed by SCOPUS or Web of Science (WOS). Besides, we also considered some good ranked conference papers. Extensive literature is available covering the deep analysis of different features and classifiers applied on EEG datasets for seizure detection [31, 34, 35]. Both, feature extraction and applying classification techniques are challenging tasks. Previous literature reveals that for the past few years, interest has been increased in the application of machine learning classifiers for extracting meaningful patterns from EEG signals, which helps for detecting seizures, its location in the brain, and other impressive related knowledge discoveries [28, 36, 37]. Three decades ago, Jean Gotman [6, 38-40], analyzed and proposed the model for effective usage of EEG signals by applying different computational and statistical techniques for automatic seizure detection. Furthermore, the research has been carried out by different signal processing methods and data science methods to provide better outcomes [27, 34, 41-47].

\section{A framework for seizure detection}

In this section, we present a pictorial framework of the model used for seizure detection from an EEG/ECoG seizure dataset, illustrated in Fig. 2. The process comprises four steps: Data Collection, Data Preparation, Applying Machine Learning Classifiers and Performance Evaluation.

\subsection{Data collection}

The initial requirement is to collect the dataset of brain signals. For this, different monitoring tools are used. Typically, the mostly used devices are EEG and ECoG, because their channels or electrodes are implanted by glue on the surface of the scalp as per 10-20 International
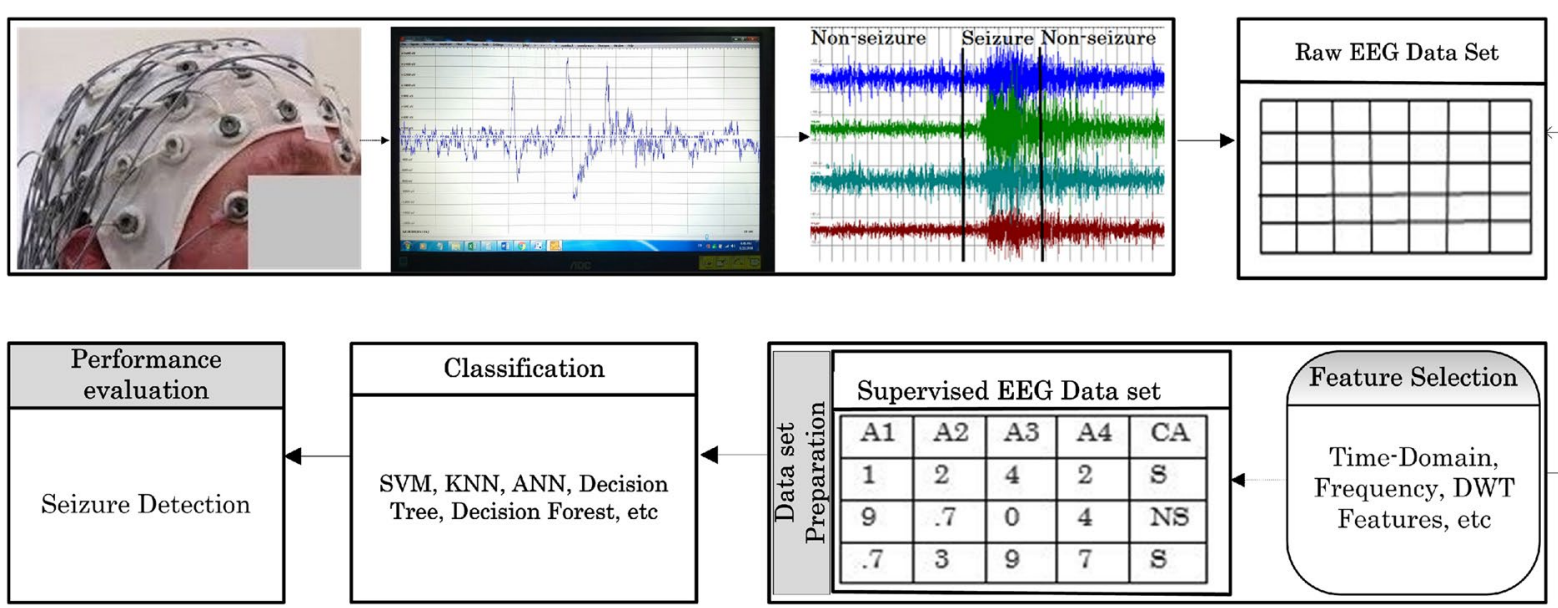

Fig. 2 Basic model of epileptic seizure detection. This explains the basic steps to collect the dataset by EEG medium, display of raw EEG signals, transform EEG signals to two-dimensional table, feature selection, prepare the dataset with seizure (S) and non-seizure (NS), apply machine learning classifier(s) and seizure detection, or other related tasks 
system [48] at different lobes. Each of them has a wire connection to the EEG device, providing timely information about the variations in voltage, along with temporal and spatial information [49]. As highlighted in Fig. 2, the EEG channels are placed on the subject's scalp, and the electrical signals are read by the EEG monitoring tool and it displays these raw signals over the screen. Further, these raw signals have been carefully monitored by the analyst and classified into 'seizure' and 'non-seizure' states.

\subsection{Data transformation}

After data collection, the next crucial step is to transform the signal data into a 2-D Table format. The reason for this is to make it easier for analysis and provide necessary knowledge like seizure detection. This datum is raw because it has not been processed yet. Therefore, it will not be suitable to give relevant information. To do the processing, different feature selection modalities have been applied. This step also presents the dataset as supervised, which means that it provides the class attribute with possible class-values.

\subsection{Dataset preparation}

For data transformation, data processing is a decisive step to extract meaningful information from the collected raw dataset. As such, different feature extraction techniques have been used; as shown in Table 1. These methods are generally applied to the extracted EEG signal dataset [31, 34]. The raw dataset becomes rich in terms of different statistical measure values.

After feature extraction processing, the dataset becomes more informative that it ultimately helps the classifier for retrieving better knowledge.

\subsection{Applying machine learning classifiers and performance evaluation}

To achieve a high accuracy of seizure detection rate and explore relevant knowledge from the EEG processed dataset, different supervised and unsupervised machine learning have been used.

\subsubsection{Classification}

In classification, a dataset $D$ has a set of 'non-class attributes', and a 'class attribute.' They are the principal components and their pertinent knowledge is very important, as both have a strong association for potential classification. The target attribute is defined as the class attribute' $C$, and it comprises more than one class values, e.g., seizure and non-seizure. On the contrary, attributes $A=\left\{A_{1}, A_{2} . A_{3} \ldots A_{n}\right\}$ are known as 'non-class attributes' or predictors $[50,51]$. The following classifiers have been popularly used in seizure detection. Common classifiers such as SVM [52], decision tree [53] and decision forest [54] are applied to the processed EEG dataset for seizure detection.

\subsubsection{Performance evaluation}

The accuracy of the obtained results is used to evaluate different methods. The most popular training approach is tenfold cross-validation [55], where each fold, i.e., one horizontal segment of the dataset is considered to be the testing dataset and the remaining nine segments are used as the training dataset $[56,57]$.

Except for the accuracy, the performance of the classifiers is commonly measured by the following metrics such as precision, recall, and f-measure [58]. These are based on four possible classification outcomes-True-Positive (TP), True-Negative (TN), False-Positive (FP), and FalseNegative (FN) as presented in Table 2.

Precision is the ratio of true-positives to the total number of cases that are detected as positive $(\mathrm{TP}+\mathrm{FP})$.

Table 1 Feature extraction methods and features used on EEG signal dataset

\begin{tabular}{|c|c|}
\hline Feature extraction methods & Relevant features \\
\hline Time-domain features & $\begin{array}{l}\text { Mean, variance, mode, median, skewness, kurtosis, max, min, zero crossing, line length, energy, power, } \\
\text { Shannon entropy, sample entropy, approximate, entropy, fuzzy entropy, hurst exponent, standard devia- } \\
\text { tion }\end{array}$ \\
\hline Frequency-domain features & Spectral power, spectral entropy, energy, peak frequency, median frequency \\
\hline Time-frequency-domain features & $\begin{array}{l}\text { Line length, min, max, Shannon entropy, approximate entropy, standard deviation, energy, median, root } \\
\text { mean square }\end{array}$ \\
\hline Discrete Wavelet Transformation (DWT) & $\begin{array}{l}\text { Bounded variation, coefficients, energy, entropy, relative bounded, variation, relative power, relative scale } \\
\text { energy, variance, standard deviation }\end{array}$ \\
\hline Continuous Wavelet Transformation (CWT) & Energy's standard deviation, energy, coefficient z-score, entropy, \\
\hline Fourier Transformation (FT) & $\begin{array}{l}\text { Median frequency, power, peak frequency, spectral entropy power, spectral edge frequency, total spectral } \\
\text { power }\end{array}$ \\
\hline
\end{tabular}


Table 2 Classification outcomes

\begin{tabular}{|c|c|c|}
\hline Acronym & Detection type & Real-world scenario \\
\hline $\mathrm{TP}$ & True-positive & If a person suffers to 'seizure' and also correctly detected as a'seizure' \\
\hline $\mathrm{TN}$ & True-negative & The person is actually normal and the classifier also detected as a 'non-seizure' \\
\hline $\mathrm{FP}$ & False-positive & $\begin{array}{l}\text { Incorrect detection, when the classifier detects the normal patient as a 'seizure' } \\
\text { case }\end{array}$ \\
\hline FN & False-negative & $\begin{array}{l}\text { Incorrect detection, when the classifier detects the person with 'seizure(s)' as a } \\
\text { normal person. This is a severe problem in health informatics research }\end{array}$ \\
\hline
\end{tabular}

This table describes each parameter metric considering seizure and non-seizure case

It is the percentage of selected cases that are correct, as shown in Eq. 1. High precision means the low false-positive rate.

$$
\text { Precision }=\frac{T P}{T P+F P} \times 100 \%
$$

Recall is the ratio of true-positive cases to the cases that are actually positive. Equation 2 shows the percentage of corrected cases that are selected.

$$
\text { Recall }=\frac{T P}{T P+F N} \times 100 \%
$$

Despite getting the high Recall results of the classifier, it does not indicate that the classifier performs well in terms of precision. As a result, it is mandatory to calculate the weighted harmonic mean of Precision and Recall; this measure is known as F-measure score, shown in Eq. 3. The false-positives and the false-negatives are taken into account. Generally, it is more useful than accuracy, especially when the dataset is imbalanced.

$$
\text { F-measure }=2 \times \frac{(\text { Precision } \cdot \text { Recall })}{\text { Precision }+ \text { Recall }}
$$

\section{Publicly available datasets}

For data scientists and researchers, a dataset used is important for evaluating the performance of their proposed models. Similarly, in epileptic seizure detection, we need to capture the brain signals. EEG recording is the most used method for monitoring brain activity. These recordings play a vital role in machine learning classifiers to explore the novel methods for seizure detection in different ways such as onset seizure detection, quick seizure detection, patient seizure detection, and seizure localization. The significance of publicly available datasets is that they provide a benchmark to analyze and compare the results to others. In the following section, we will describe the popular datasets that are widely used on epilepsy.

\subsection{Children Hospital Boston, Massachusetts Institute of Technology-EEG dataset}

This dataset is publicly available on a physionet server and prepared at Children Hospital Boston, Massachusetts Institute of Technology (CHB-MIT) [59, 60]. It can be collected easily via Cygwin tool which interacts with the physionet server. It contains the number of seizure and non-seizure EEG recordings for each patient of the CHB [61]. The dataset comprises 23 patients; 5 males, aged 3-22 years, and 17 females aged 1.5-19. Each patient contains multiple seizure and non-seizure recording files in European data format (.edf), representing the spikes with seizure start and end time, which is easily visible at a browser called an 'EDFbrowser.' The primary datasets are in the 1-D format, containing EEG signals that are obtained through the different types of channels that were placed on the surface of the brain as per 10-20 International System. All these signals of the dataset were sampled at the frequency of $256 \mathrm{~Hz}$.

\subsection{ECoG Dataset, Epilepsy Centre, University of California}

This is a publicly available dataset of electrocorticogram (ECoG) signals from an epileptic patient, which was collected from the Epilepsy Center, University of California, San Francisco (UCSF) [62]. It was originally collected by implanting 76 electrodes on the scalp in both invasive (12-electrodes) and non-invasive manner (64-electrodes). It comprises 16 files altogether. Out of these, eight files $(F 1, F 2, \cdots F 8)$ are classified as 'pre-ictal' meaning the stage before the seizure. The rest of the files $(F 9, F 10, F 11, \cdots F 16)$ represent the 'ictal' stage data. The collected data are sampled at the frequency of 400 $\mathrm{Hz}$ (i.e., 400 cycles/s) and the total duration is $10 \mathrm{~s}$. As a result, there are $(400$ cycles/s $\times 10 \mathrm{~s}) 4000$ cycles in each file [63].

\subsection{The Freiburg-EEG dataset}

This dataset was collected from the invasive EEG recordings of 21 patients ( 8 males aged 13-47 years, 13 females aged 10-50 years) suffering from medically intractable focal epilepsy. It was recorded during an invasive presurgical epilepsy monitoring at the Epilepsy Centre of 
the University Hospital of Freiburg, Germany [64]. Out of 21 patients, 13 patients had $24 \mathrm{~h}$ of recordings, and 8 patients had less than $24 \mathrm{~h}$. These recordings are interictal, and together they provide 88 seizures.

\subsection{Bonn University-EEG dataset}

The dataset comprises five subsets, where each one denoted as (A-E) contains 100 single-channels recording, and each of them has a $23.6 \mathrm{~s}$ duration, captured by the international 10-20 electrode placement scheme. All the signals are recorded with the same 128-channel amplifier system channel [65].

\subsection{BERN-BARCELONA-EEG dataset}

This dataset comprised EEG recordings derived from five pharmacoresistant temporal lobe epilepsy patients with 3750 focal and 3750 non-focal bivariate EEG files. Three patients were seizure-free, with two patients only having auras but no other seizures following surgery. The multichannel EEG signals were recorded with an intracranial strip and depth electrodes. The $10-20$ positioning was used for the electrodes' implantation. EEG signals were either sampled at 512 or $1024 \mathrm{~Hz}$, depending on whether they were recorded with more or less than 64 channels. According to the intracranial EEG recordings, they were able to localize the brain areas where seizures started for all five patients [66]. This dataset is good for the seizure localization purpose.

\section{Seizure detection based on statistical features and machine learning classifiers}

This section explains the comprehensive detail of work on seizure detection using statistical features, classifiers-'black-box' and 'non-black-box'. They are illustrated in Table 3. In brief, the 'black-box' classifiers are those which provide the accuracy without mentioning the reasons behind the results such as ANN and SVM [67]. They are unable to explain their classification steps. Whereas, 'non-black-box' classifiers such as decision forest and random forest can able to explain each step of the processing, which is human-understandable. As a result, it helps in human-interpretable knowledge with high accuracy [68].

\subsection{Seizure detection based on statistical features}

If we apply machine learning classifier(s) directly to raw EEG/ECoG datasets, it may not produce enough sensible patterns. Therefore, selecting significant and capable statistical features from EEG and ECoG raw datasets is one of the challenges and a crucial task. The nature of EEG and ECoG signals is very complex, non-stationary and time-dependent [105-107]. As such, we can apply the machine learning classifier(s) to the processed datasets, which will ultimately assist to solve various neurological problems; for example, identifying seizure's stages, accurate seizure detection, fast detection, etc. In Table 3, we summarize a review of several studies.

The significant statistical features were extracted by different types of transformation techniques; discrete wavelet transformations (DWT), continuous wavelet transformation (CWT), Fourier transformation (FT), discrete cosine transformation (DCT), singular value decomposition (SVD), intrinsic mode function (IMF), and time-frequency domain from EEG datasets [34, $71,79,108$ ]. Logesparan et al. [34] used different types of feature extraction methods for seizure detection, but they reported that two features-'line length' and 'relative power'-are the good performers for seizure detection. Guerrero-Mosquera [109] applied three time-domain features-line length, frequency, and energy on the raw EEG dataset. These features claim to be suitable for seizure detection and other brain-related applications such as computer interface (BCI). The claimed performance was evaluated using the following metrics such as sensitivity, specificity, F-score, receiver operating characteristics (ROC) curve, and percentile bootstrap measures. Duo Chen [84] used DWT with the SVM classifier on two benchmark datasets-CHB-MIT and Bonn University, achieved seizure detection accuracies of $92.30 \%$ and 99.33\%, respectively. Ramy Hussein et al. [100] proposed a new featured L1-penalized robust regression (L1PRR) for seizure detection, the issue with their approach is computational complexity. Zavid and Paul [99] focused on classifying the 'ictal' and 'inter-ictal' states, where they used four features DCT, DCT-DWT, SVD, and IMF; the obtained signals are further classified by LS-SVM due to less computational cost.

Several researchers have contributed to seizure detection using a single feature $[108,110]$. The feature line length' $[108,110]$ was applied to an EEG dataset; approximately $4.1 \mathrm{~s}$ of mean detection latency is recorded at a false alarm rate of $0.051 \mathrm{Fp} / \mathrm{h}$. Further, Guo et al. [69] also used 'line length' but with the ANN for classifying the records obtained by EEG signals. Their automated seizure detection accuracy is $99.6 \%$. A system was proposed by Koolen et al. [70] to detect seizures from EEG recordings. This detection system uses a single feature-'line length' The performance of this system shows $84.27 \%$ accuracy, $84.00 \%$ sensitivity and $85.70 \%$ specificity, which are comparatively lower than the results of Guo et al. [69].

After 3 years of study on several of statistical features [34], Logesparan et al. [71] proposed the 'line length' feature for normalization and discrimination of class values from EEG datasets. It is noted that 'line length' could be taken as the strongest feature and provides considerable 
Table 3 Overview of existing work on seizure detection using-machine learning classifiers, features, performance score, performance metrics, datasets, and Authors

\begin{tabular}{|c|c|c|c|c|c|}
\hline Classifier(s) & Feature(s) & Performance (\%) & Performance metrics & Dataset & Authors \\
\hline SVM & Vector & 96 & Sensitivity (Sen) & CHB-MIT & Shoeb and Guttag [41] \\
\hline Random forest & Time and frequency & 93.8 & Senstivity & EPILEPSIAE & Donos et al. [44] \\
\hline ANN & Line length & 99.6 & $\begin{array}{l}\text { Classification accuracy } \\
\text { (Class Acc) }\end{array}$ & BONN & Guo et al. [69] \\
\hline Burst detection algo & Line length & $84.27,84,85.7$ & $\begin{array}{l}\text { Acc, Sen, Specificity } \\
\text { (Spec) }\end{array}$ & NICU, Belgium & Koolen et al. [70] \\
\hline Normalization & Line length & 52 & $\mathrm{ROC}$ & CHB-MIT & Logesparan et al. [71] \\
\hline ELM and BPNN & SE & 95.6 & Class Accuracy & BONN & Song and Lio [72] \\
\hline SVM and ELM & $\mathrm{AE}$ and $\mathrm{SE}$ & 95.58 & Class Accuracy & BCI Lab, Colarodo & Zhang et al. [73] \\
\hline SVM & DWT & 94.8 & Avg Accuracy & CHB-MIT & Ahmad et al. [74] \\
\hline GMM & $\begin{array}{l}\text { Spectral, hybrid, } \\
\text { temporal }\end{array}$ & 87.58 & Avg Accuracy & CHB-MIT & Gill et al. [75] \\
\hline Random forest & PCA, STF, Moving Max & $97.12,99.29,0.77 / \mathrm{h}$ & Sen, Spec, FPR & CHB-MIT & $\begin{array}{l}\text { Orellana and Cerqueira } \\
\text { [76] }\end{array}$ \\
\hline $\begin{array}{l}\text { Random forest and } \\
\text { k-NN }\end{array}$ & Spectral power & $\begin{array}{l}80.87,47.45,2.5 / \mathrm{h} \\
56.23\end{array}$ & Sen, Prec, FPR, F-meas & CHB-MIT & Birjandtalab et al. [77] \\
\hline Boosting & Stockwell & $94.26,96.34$ & Sen, Spec & Freiburg & Yan et al. [78] \\
\hline $\begin{array}{l}\text { SVM, MLP, KNN, Naïve } \\
\text { bayes }\end{array}$ & Energy & 98.75 & Class Acc & EPILEPSIAE & Amin et al. [79] \\
\hline Random forest & Entropy and DWT & 98.45 & Class Acc & BONN & Mursalin et al. [80] \\
\hline SVM & Time-Frequency & $90.62,99.32$ & Sen, Spec & CHB-MIT & Zabihi et al. [81] \\
\hline Random forest & Time-domain & 96.94 & ROC curve & Kaggle & Truong et al. [82] \\
\hline $\begin{array}{l}\text { SVM, LDA, QDA, LC,PC, } \\
\text { DT, KNN, UDC, } \\
\text { PARZEN }\end{array}$ & Time-frequency & 84,85 & Sen, Spec & CHB-MIT & Fergus et al. [83] \\
\hline SVM & DWT & 86.83 & Confusion Matrix & CHB-MIT & Chen et al. [84] \\
\hline $\begin{array}{l}\text { SVM and neural } \\
\text { network }\end{array}$ & DWT and CWT & 99.1 & Overall Acc & BONN & Satapathy et al. [85] \\
\hline ELM & Time-frequency & $97.73,0.37 / \mathrm{h}$ & Sen, false alarm rate & Freiburg & Yuan et al. [86] \\
\hline SVM & DWT & 99.38 & Class Acc & BONN & Subasi et al. [87] \\
\hline LS-SVM & FFT and DWT & 100 & Class Acc & BONN & Al Ghayab et al. [88] \\
\hline SVM and Naïve bayes & $\begin{array}{l}\text { Entropy, RMS, variance, } \\
\text { energy }\end{array}$ & $96.55,95.63,95.7$ & Sen, Spec, Acc & CHB-MIT & Selvakumari et al. [89] \\
\hline LS-SVM & 8 types of Entropies & $100,99.4,99.5$ & Sen, Spec, Acc & BONN & Chen S et al. [90] \\
\hline ANN & Spectral power & 86 & F-meas & CHB-MIT & Birjandtalab et al. [91] \\
\hline KNN and GHE & - & 100 & Class Acc & BONN & Lahmiri and shumel [92] \\
\hline Random forest & DWT & $99.74,0.21 / \mathrm{h}$ & Sen, FPR & BONN and Freiburg & Tzimourta et al. [93] \\
\hline Random forest & $\begin{array}{l}\text { STFT, mean, energy and } \\
\text { std dev }\end{array}$ & 96.7 & Class Acc & BONN & Wang et al. [94] \\
\hline $\begin{array}{l}\text { Random forest, SVM, } \\
\text { KNN, and Adaboost }\end{array}$ & $\begin{array}{l}28 \text { statistical and time- } \\
\text { frequency features }\end{array}$ & $\begin{array}{l}97.6,94.4,96.1,92.9 \\
98.8,0.96\end{array}$ & $\begin{array}{l}\text { Sen, Spec, Acc, PPR, } \\
\text { NPR, ROC }\end{array}$ & Bern-Barcelona & Raghu and Sriraam [95] \\
\hline $\begin{array}{l}\text { ANN,KNN,SVM, and } \\
\text { Random forest }\end{array}$ & $\begin{array}{l}\text { Mean, std dev, power, } \\
\text { skewness, kurtosis, } \\
\text { absolute mean }\end{array}$ & 100 & Overall Accuracy & Freiburg and CHB-MIT & Alickovic et al. [96] \\
\hline SVM & Energy & 99.5 & Class Acc & BONN and Barcelona & Fasil and Rajesh [97] \\
\hline $\begin{array}{l}\text { SVM and Random } \\
\text { forest }\end{array}$ & 10-time and frequency & 0.98 & $\operatorname{ROC}(A \cup C)$ & EPILEPSIAE & Manzouri et al. [98] \\
\hline LS-SVM & $\begin{array}{l}\text { DCT, SVD, IMF, DCT- } \\
\text { DWT, }\end{array}$ & 91.36 & Acc, Sen, Spec & Freiburg & Parvez and Paul [99] \\
\hline SysFor and Forest CERN & 9 statistical features & 100 & Class Acc & Epilepsy Centre UCSF & Siddiqui et al. [63] \\
\hline Random forest & $\begin{array}{l}\text { L1-penalized robust } \\
\text { regression (L1PRR) }\end{array}$ & 100 & Class Acc & BONN & Hussein et al. [100] \\
\hline
\end{tabular}


Table 3 (continued)

\begin{tabular}{llllll}
\hline Classifier(s) & Feature(s) & Performance (\%) & Performance metrics & Dataset & Authors \\
\hline $\begin{array}{l}\text { SVM, NB, KNN, random } \\
\text { forest, logistic model }\end{array}$ & 15-features & $97.40,97.40,97.50$ & Acc, Sen, Spec & BONN & Mursalin et al. [101] \\
$\begin{array}{l}\text { Trees (LMT) } \\
\begin{array}{l}\text { Random forest } \\
\text { ANN }\end{array}\end{array}$ & IMF & $98.4,98.6,96.4$ & Sen, Spec, Acc & BONN & Sharma et al. [102] \\
$\begin{array}{l}\text { Decision forest-Ran- } \\
\text { dom forest, Boosting }\end{array}$ & 9 statistical features & $96.67,74.36,84.06$ & Pre, Rec, F-measure & CHB-MIT & Tzallas et al. [103] \\
\hline
\end{tabular}

output. Based on previous studies, the 'line length' can be taken with other features, and the result would be more promising, specifically in machine learning. This is because the dataset dimension would also increase with meaningful statistical information in the attributes.

Some other studies on seizure detection based on a single feature, i.e., entropy and its sub-types such as approximate entropy (AE) and sample entropy (SE), have also been done $[45,72,73,111]$. The entropy feature helps to find the random behaviour of EEG signals and takes depth benefits in measuring the impurity of the signals $[112,113]$. The entropy feature has been used widely where data are in the form of signals such as ECG, [114], EEG, and ECoG [36]. This helps in further steps of the detection model.

Acharya et al. [111] used four different types of entropy-based features: sample entropy, approximate entropy, phase entropy (S1), and phase entropy (S2) of the EEG datasets. The processed dataset from these entropy features was used for seizure detection. In another study, Chen et al. [90] used eight different kinds of entropy feature-approximate, sample, spectral, fuzzy, permutation, Shannon, conditional and correction conditional on a raw EEG dataset; further, the processed data were classified into three class values: 'ictal', 'inter-ictal' and 'normal stage', and their accuracy is $99.50 \%$. A tool was proposed by Selvakumari et al. [89] using four features-entropy, root mean square (RMS), variance, and energy. Based on these features, the detection was done using SVM and naïve Bayesian classifiers with a reported accuracy of $95.63 \%$. The tool is also able to find the seizure region in the brain; however, they did not mention the exact percentage of seizure location. Song and $\mathrm{Li}$ [72] built classification models by two classifiers-Extreme Learner Machine (ELM) and the back-propagation neural network (BPNN). Overall, their findings show 95.6\% of classification accuracy with less execution time. Yong Zhang et al. [73] applied two entropy features-AE and SE on two different classifiers-ELM and SVM for processing EEG dataset. The SE features with ELM provide good classification accuracy compared to the $\mathrm{AE}$ feature whilst detecting the seizure.
The energy feature has been significantly used in seizure detection [115]. It plays a vital role particularly when the seizure is detected by the epoch- or windows-based method. This means that the EEG signals are divided into various segments $[79,94]$. An exponential energy feature has been introduced by Fasil and Rajesh [97], which helps in identifying the irregularities in amplitude EEG signals.

Observations This section has provided an overview of the contributions of statistical features to seizure detection and their importance. Some researchers detect seizures using multiple sets of features, whilst others select a single feature such as 'line length'. We recommend the 'line length' feature to be in the list of the set of suitable features for seizure detection because it is helpful in measuring the EEG signals complexity. It plays a sensitive role in the changes at the frequency and amplitude of signals. As a result, it helps to discriminate against the 'seizure' and 'non-seizure' cases. However, from the data science point of view, it is very important to see the various perspectives of each brain signals by observing other statistical features. Furthermore, we also suggest not to use the irrelevant feature(s) as they will unnecessarily increase the dataset size which results in an increase in computational time and gives insensible patterns too. As a result, it becomes a hassle to machine learning classifiers and users rather than providing the benefit. Some researchers $[95,98,101]$ used a large number of features, which increases the attribute size, and results in more computational time and less accuracy. So, if we take the fewer features as previous researchers have done $[71,73$, 79] this will give the low-dimensional dataset, which will not be fruitful for the knowledge discovery process. The next section illustrates the seizure detection by 'blackbox' classifiers. As far as the classification purpose is concerned, it would be better to take more relevant statistical features, which can be integrated into knowledge discovery and a good performance rate.

\subsection{Seizure detection based on black-box classifiers}

The classifiers such as SVM, ANN, and KNN are considered as prominent ones due to their remarkable performances in different domains $[67,116]$. Each technique 
has its pros and cons, and 'black-box' methods are not an exception to this [104]. Even though these classifiers contribute well to brain datasets, some of the relevant works on seizure detection using these classifiers are reported here.

The study of Satapathy et al. [85] was based on two 'black-box' approaches-SVM and Neural networks using different kernel methods for seizure detection against a large EEG dataset. The performance of each classifier is measured independently by the majority voting system, and it was found that SVM was more capable than other neural networks. Subasi et al. [87] proposed the solution to detect seizure using a hybrid approach of SVM, genetic algorithm (GA), and particle swarm optimization (PSO). The method achieved impressive accuracy, i.e., $99.38 \%$, but the problem is that the classifier trains the dataset twice, one for SVM-GA and another for SVM-PSO. This could be a time-consuming.

Shoeb and Guttag [41] performed seizure detection on their arranged dataset of Child Hospital Bostan, MIT (CHB-MIT) [60] using SVM with the vector feature and achieved the estimated accuracy of $96 \%$. Dorai and Ponnambalam [42] came with an idea of the epoch, which means dividing the dataset into smaller time frames. Further, they applied an ensemble of four 'blackbox' approaches-LDA, KNN, CVE, and SVM on these epoch EEG datasets. This approach provides the prediction of onset seizures $65 \mathrm{~s}$ earlier. Classifying the EEG data into two class ' 'seizure" and 'non-seizure', Birjandtalab et al. [117] used a Gaussian mixture model (GMM) before detecting the seizure, and obtained 90\% accuracy with $85.1 \% \mathrm{~F}$-measure. They also raised the issue of class imbalance in their dataset. Tzallas et al. [103] used time-frequency-domain features with ANN for the EEG dataset and obtained 100\% accuracy for the 'seizure' and 'non-seizure' classification problem; with epochs' datasets the accuracy is $97.7 \%$ from $(A, B, C$, and $D)$ for 'nonseizure' and set E for 'seizure' epoch classes. Amin et al. [79] extracted relative energy features from the DWT method, and four classifiers-SVM, MLP, KNN, and Naïve Bayes-were applied for the classification purpose, the result shows $98 \%$ of SVM accuracy, which outperforms remaining classifiers. A framework had been proposed by K. Abualsaud et al. [118] using the ensemble of 'black-box' classifiers for automated seizure detection on noisy EEG signals, and the reported classification accuracy is $95 \%$. However, the ensemble approach did not provide good accuracy as desired because all four classifiers were 'black-box'.

In 2018, Lahmiri et al. [92] used generalized Hurst exponent (GHE) and $\mathrm{KNN}$, to propose a system for identifying the 'seizure' and 'non-seizure' classes from intracranial EEG recordings, detection rate, with 100\% accuracy rate. Further, Lahmiri et al. [43] exploited GHE with SVM, to classify the 'seizure' and 'non-seizure', and also they found $100 \%$ accuracy in less time. Here, the good indication is that authors claim the good accuracy in less time for seizure detection. But, the authors did not clearly define how many times the seizure can be detected. In another study by $\mathrm{Al}$ Ghayab et al. [88], the obtained accuracy is $100 \%$ as a result of using the concept of Information gain theory, to extract and rank the meaningful features from EEG signal dataset. The least square-support vector machine (LS-SVM) is then applied to classify the seizure cases. Moreover, due to the "blackbox"s nature of applied classifiers, the authors could not explore any other related aspects in terms of Knowledge discovery. Zabihi et al. [81] did patient-specific seizure detection using SVM classifier on the processed dataset with a good set of features, comprising time-domain, frequency-domain, time-frequency domain, and non-linear feature. The performance of their model has achieved an average of $93.78 \%$ sensitivity and a specificity of $99.05 \%$. Here, it is noteworthy that they skip an important feature-'line length', from the available literature, which is prominently used in seizure detection. We also argue that CHB-MIT dataset [60] is imbalanced because, in an hour(s) of recording, a seizure time span is for a few seconds.

\section{Observations}

The main issue with 'black-box' classifiers is that they only make prediction without providing logic rules or patterns. That is why, they are not recommended for extracting sensible knowledge. For example, for class imbalance issues in EEG datasets, insufficient related literature is found, and the researchers who attempted to work on this problem did not provide a conceivable solution as to how to solve the class imbalance issue whilst detecting the seizure.

\subsection{Seizure detection based on non-black-box classifiers}

'Black-box' classifiers are unable to express their classification procedure for human interpretation $[67,104,116]$. Consequently, there are fewer chances for knowledge discovery and better accuracy performance. Therefore, the concept of 'non-black-box' classifiers such as decision trees, and decision forests came into practice.

Chen et al. [119] first introduced the decision tree to the EEG dataset for seizure detection. Kemal and Saleh [120] used a C5.0 decision tree [121] algorithm to explore the logic rules for seizure detection, with an average accuracy of $75 \%$. When the same C5.0 was applied to the same dataset processed by Fourier transformation the obtained accuracy with cross-validation was, however, 98.62\%. A few related works are been available, where only a decision tree method is applied seizure detection 
because of less accuracy and a limited number of patterns obtained from the logic rules of a decision tree [122]. As a result, both the knowledge discovery and accuracy suffer. However, this gap can be filled by applying decision forest approaches instead [51, 57, 123].

Through the literature, it is found that the decision forest approaches are more effective than the single decision tree $[57,124]$, because the decision tree often gives a confined set of rules and overfitting issue is also raised [68]. The rules are extracted from training data by a decision tree that generates either limited or a single set of logic rules (Say, wherever C2_Entropy value $\leq 101.01$ then Class_value $=$ seizure $)$ and stops growing the tree further records in the training dataset once the rule is accepted. However, if we generate a decision forest on the training data, we can achieve multiple sets of decision trees with the combination of sensible logic rules and a higher accuracy rate due to the majority voting method [57]. Decision forest classifiers $[54,68]$ are the type of ensemble methods that are used frequently. These are also used in seizure detection as they provide a high accuracy rate which depends on the majority voting method from the ensemble of decision trees. Moreover, they produce more logic rules as multiple decision trees from the training data $(D)$ [123]. These logic rules are humanly interpretable, and data scientists can easily interrelate them with other seizure-related information from EEG datasets.

Siddiqui and Islam [125] used Systematic Forest (SySFor) to detect the seizure on ECoG without epoch reduction. Further, Siddiqui et al. [63] applied two decision forests-Systematic Forest (SysFor) [123] and Forest CERN [51] on nine statistical features for quick seizure detection using the concept of epoch length reduction. It is based on dividing the size of training dataset $D$ into $D_{1}, D_{2}, \ldots D_{n}$ and testing the accuracy at every epoch of the dataset. These sub-datasets are in descending order in terms of time duration. If the seizure can be detected in a shorter epoch length without a decline in accuracy, then we can use the same one, which results in fast seizure detection. They achieved $100 \%$ accuracy. The limitation of this work is that authors have taken the dataset of a single patient, this could be tested for more patients. Several researchers have taken the advantages of random forest classifier for detecting the seizures [76, 78, $82,126]$. Because researchers/data scientists are able to see the logic rules and interpret them correspondingly. Moreover, it also provides good accuracy $[44,76-78,80$, 82]. Donos et al. [44] applied decision forest classifierrandom forest, on time and frequency domains' feature, which was extracted from an IEEG (Intra-cranial EEG) dataset. It helped in selecting the intra-cranial channels for early seizure detection in a closed-loop circuit. The results claimed that the system can detect the seizure with 93.8\% sensitivity. Wang et al. [94] developed the greedy approach of random forest, i.e., forest-grid search optimization (RF-GSO), with this method and they found 96.7\% accuracy. The shortcoming of this technique is that the performance could decline if EEG signals are too noisy. Tzimourta et al. [93] applied random forest to monitor seizure activities on the two benchmark epilepsy datasets [64, 65], the reported performance is $99.74 \%$. Pinto-Orellana and Fábio R. Cerqueira [76] also used the random forest on the processed CHB-MIT dataset by a Spectro-temporal feature, and 70s, and the accuracy of each block is $98.30 \%$.

Truong ND et al. [82] had carried out novel work of channel selection whilst detecting the seizure. Their key contribution is that they also focus on channels contributing mostly to automatic seizure detection. They used the random forest to solve channel selection and seizure detection, and which achieving 96.94\% area under the curve (AUC). In another work, Mursalin et al. [80] proposed a method for seizure detection by selecting features with an Improved Correlation-based Feature Selection(ICFS). Basically it is a fusion of time and frequency domain. Then, a random forest classifier was applied for the seizure detection model. The obtained average classification accuracy by this approach was $98.75 \%$.

Some other works have used an ensemble of 'nonblack-box' classifiers such as boosting, bagging and random subspace [78, 127]. Yan et al. [78] applied a boosting classifier achieving $94.26 \%$ of accuracy, although the results were not as impressive as the ones obtained by [44], which used a random forest classifier. Hosseini [128] used Random subspace classifier along with an SVM classifier, to classify and detect seizures. Here, the benefit of applying a subspace on big datasets is to divide them into sub-datasets based on the random subspace concept, and then the SVM classifier was applied to each sub-dataset. Ensemble accuracy (EA) was calculated by the majority voting method, which was 95\%. Apart from this study, the same authors of Hosseini et al. [126] recently did another research using an ensemble of classifiers. First, they created bootstrap samples using a random subspace method, and then applied classifiers such as SVM, KNN, extended nearest neighbor (ENN), and multilayer perceptron (MLP) obtaining 97\% accuracy. Hussein et al. [100], proposed a novel feature extraction method, i.e., L1-penalized robust regression (L1PRR), which uses three common symptoms during seizures-muscles artifacts, eyes movement, and white noise. Inputting these features help the random forest classifier to obtain 100\% accuracy.

Observations In comparison to decision trees, decision forest classifiers are tremendously used on brain datasets 
for exploring different research goals. It is difficult to suggest a particular classifier whilst dealing with a highdimensional dataset, but a random forest classifier can be a capable classifier. However, it also criticizes that not all the 'non-black-box' classifiers are peculiar to detect seizures and have also pointed out the objection on the drawback of using a single decision tree classifier.

\subsection{Seizure detection based on black-box and non-black-box machine learning classifiers}

From the literature, it is found that just a single machine learning classifier is not sufficient. Therefore, to take advantage of both 'black-box' and 'non-black-box' classifiers, some researchers utilized them in their experiments. This section provides a comprehensive review of classifiers applied together to detect the seizure.

Acharya et al. [111] used the ensemble of seven different classifiers-Fuzzy surgeon classifier (FSC), SVM, KNN, Probabilistic neural network, GMM, decision tree and Naïve Bayes for distinguishing the three states of a patient as 'normal, 'pre-ictal' and 'ictal'. The overall accuracy is $98.1 \%$. Fergus et al. [83] also used distinct classifiers such as linear discriminant analysis (LDA), quadratic discriminant classifier (QDC), logistic classifier, uncorrelated normal density-based classifier (UDC), polynomial classifier, KNN, PARZEN, SVM, and decision tree on the processed data with seven features such as entropy, RMS, skewness, and variance. They contributed that the detected patient is suffering from a 'Generalize seizure' (means affecting whole brain region) across different patients without prior information about the seizure focal points. Mursalin et al. [101] proposed a method to reduce the data size, statistical sampling technique called optimum sample allocation technique, and to reduce the features they develop a feature selection algorithm. The analysis was done on the combination of five classifiersSVM, KNN, NB, Logistic Model Trees (LMT) and Random forest.

Rand and Sriram [95] used four classifiers such as SVM, KNN, random forest, and Adaboost on a highdimensional dataset prepared by 28 features. Their result shows that SVM outperforms on the cubic kernel. In another study, Manzouri et al. [98] used SVM and random forest on the dataset produced by 10-time and frequency features. In comparison to SVM-based detector, random forest classifier outperforms. Subasi et al. [96] achieved $100 \%$ of accuracy using four machine learning classifiers such as ANN, KNN, SVM, and random forest on two popular datasets-Freiburg and CHB-MIT to classify the three different states of seizures 'pre-ictal', 'ictal', and 'inter-ictal'. Sharma et al. [102] proposed an automated system using iterative filtering and random forest for classifying the EEG signals. This work achieved classification accuracies of $99.5 \%$ on BONN dataset (A-E), for A versus $E$ subsets, $96 \%$ for $D$ versus $E$ subsets, and $98.4 \%$ for $A B C D$ versus $E$ classes of EEG signals. Birjandtalab et al. [77] used two classifiers for different purposes; KNN is used to discriminate the 'seizure' and 'non-seizure' classes, whereas random forest is used to explore the significant channels. Here, the random forest also helps in the dimension reduction problem. The main benefit of selecting suitable channels is that it helps in providing relevant required information from the chosen channels, and reduces the computational cost of a classifier too. However, the authors did not mention here the important information from channel selection like finding the seizure location from the brain scalp. The main critic in $[95,98,101]$ is that because of a large number of features, the attribute size of dataset will increases, and as a result the accuracy and computation time suffer.

\subsubsection{Observations}

We observe that some work used an ensemble of distinguished classifiers to take the benefits separately. For example, influential channel selection can be independently done using decision forest classifiers like a random forest. But authors used other classifiers such as SVM and KNN for classifying the seizure records with good accuracy.

\section{Seizure localization}

After a successful seizure detection, localization is an essential task for epileptic surgery [129-131]. Typically, localized seizures can be cured by surgery which arises either from the left or right region of the brain. The seizure monitoring tools such as ECoG and EEG are prominently helpful to identify the seizure location. The electrodes/channels are implanted in a non-invasive (for EEG) and an invasive manner (for ECoG). Their positioning is based on the $10 / 20(10-20)$ International system, which helps in identifying the seizure location [132]. The concept of seizure localization means identifying the region of the brain affected by a seizure. Though some types of seizures such as 'tonic-clonic' are cured by anti-epileptic drugs (AED), patients with partial seizures in some cases might go for surgery [13]. To solve this problem, finding the seizure location is an essential and challenging task for neurologists and neurosurgeon [129, 130]. The surgical target is to find a point/location/focal area from where a seizure is originating. The $10-20$ positioning system gives some clues for identifying the location of a seizure. Recently, computational and machine learning methods have been applied to identify a seizure location [130, 133].

Acar et al. [133] used trucker and non-linear multi-way Trucker kernels, and claimed that other classifiers such as 
SVD and principal component analysis (PCA) were unable to localize a seizure. Ghannad-Rezaie [134] applied an advanced swarm intelligence algorithm to seizure data for finding seizure location. Their study produced some appreciable results, and explored whether the patient's temporal lobe was affected by a seizure or not. They also suggested that SVM might be able to detect the seizure location. Moreover, they also focused on the reduction of ECoG electrodes. Mansouri et al. [135] proposed an algorithm for Seizure localization, which was tested on $10 \mathrm{sec}$ of EEG dataset from Karuniya University. Here, they have taken the small-size dataset, because recording usually takes several hours. If they had tested on a big dataset, it would have been much better. Fakhraei et al. [130] calculated the sensitivity of each region of the brain. The confident prediction rate (CPR) was compared with the AUC of ROC plots obtained by six classifiers from the dataset of 79 patients (31 males, 48 females) with 197 medical features. The study found that CPR was more suitable than ROC. They also explored that 43 patients had the temporal lobe epilepsy (TLE) on their left sides whilst 36 patients had it on the right sides of their brains. Likewise, Rai et al. [136] proposed a method for identifying the focal points of the seizure by applying two entropy-based features-'renyi entropy' and 'negentropy' with the neural network classifier. Siddiqui et al. [63] localize the seizure using two decision forest classifiers, and their results showed that the left hemisphere of a brain was more affected by the seizures.

\section{Observation}

It is found that compared to seizure detection, machine learning classifiers have not been extensively applied for seizure localization. But some literature exist on this problem. In these reported works, authors did not mention the percentage of the affected region of the brain by a seizure, and they were not able to identify the exact location at the lobes such as occipital, frontal, parietal left and parietal right. Although, it is not our primary objective in this review paper, whilst discussing the related published research, we found some interesting clues for seizure localization.

\section{Problems identified in existing literature}

One of the most significant and decisive steps is to select suitable statistical features because each channel or electrode implanted on the brain provides different statistical measures. Undoubtedly, earlier researchers made their consistent efforts to find the best features. Whilst some researchers used many features [34, 79], the others applied a few features [31, 36, 108, 112, 137] for detecting the seizure. As a data scientist, it is very important to see the different statistical perspectives of each brain signal by analyzing the statistical properties of the features such as entropy, energy, and skewness. And we must not focus on taking irrelevant feature(s) as such since it will unnecessarily increase the dataset size. Consequently, it will be more a burden to machine learning classifiers than a benefit, and if we take few features as previous researchers did $[71,73,79]$, this will give the low-dimensional dataset and it will not be beneficial for an effective knowledge discovery process. Therefore, we should select those potential features that can to provide logical results. Hence, it is advisable to select a group of features to avoid a burden to the machine learning classifiers and to get help in related knowledge discovery.

Each classifier has its own merits and demerits, depending on the dataset attributes and requirements [138]. In general, it is very difficult to point out which classifier was the most effective for brain datasets. To identify the capable classifier, several classifiers have been tested on EEG datasets and their performance has been evaluated, and the one which performs well is to be considered in solving seizure detection and imparting knowledge discovery. The literature reveals that previous researchers had applied different approaches, most of which were from 'black-box' such as ANN, KNN and SVM. The biggest shortcoming in them is that they are unable to provide the appropriate explanations for patterns and the logic rules hidden inside the models. That is why, they are not suggested for remarkable knowledge discovery process. Data scientists may not explore the internal processing of patterns [51, 104]. However, from the literature, it is noted that the 'non-black-box' approach, especially, random forest, is widely used for seizure detection $[44,76,77]$, because of its nature of generating bootstrap samples [124, 139] whilst building a decision forest. An analysis has been done to estimate the performance of machine learning classifiers on EEG datasets and has been found that ensemble non-blackclassifiers performs effectively [104]. We argue that the random forest is based on bootstrap samples and it misses some influential attributes, because it randomly selects the attribute and sometimes generates the same set of logic rules also. As a result, sometimes, it creates irrelevant information too. To overcome this issue, we also suggest some other decision forest algorithms such as SysFor [123] and Forest CERN [51] methods in seizure detection.

All these findings on seizure detection raise few interesting research questions such as selecting suitable statistical features and machine learning classifiers to take less computation time as dataset has a high volume with high dimension, and the most significant missing information from machine learning classifiers is locating the accurate point of seizure at the brain lobe(s). 


\subsection{Class imbalance issue in seizure detection}

Class imbalance is one of the serious problems [140] in machine learning and the majority is seen in medical datasets [141], particularly in EEG signals. This is because the duration of EEG recording is long, time-consuming and seizure duration is for a few seconds, which results in being prone to errors [91]. As a result, the dataset becomes highly imbalanced. Previous researchers have focused on seizure detection. Over the last few years, researchers have been focusing on the class imbalance challenge whilst detecting the seizures, and attempting to solve it by applying different conventional approaches with some novelties. Javad Birjandtalab et al. [91] used ANN with a weighted cost function to imbalanced EEG dataset, by achieving 86\% F-measure. El Saadi et al. [142] obtained $97.3 \%$ accuracy using the under-sampling method with the SVM classifier. In another work by Saadullah and Awais [143], they used a combination of SMOTE and RUSTBOST techniques for detecting seizure to imbalance seizure data with $97 \%$ accuracy. However, the research done by Yuan Qi et al. [86] was very close to the satisfactory result as they assigned the heavy weights to a minority class of the data to maintain the effective balance and solved the biasing issue. The main critique of this work is that the authors did not mentioned what weights were assigned and what was their threshold level? Here, we argue that despite of EEG data are highly imbalanced as a result of their long-hour EEG recordings, the recordings continue until the seizure is detected. The seizure(s) time spans from only seconds to minute(s). Although researchers [76, 86, 117, 143] made their efforts in addressing this issue using both 'blackbox' and 'non-black-box' classifiers, they did not propose any justifiable solutions, in terms of how big weights should be assigned to the minority (seizure) classes.

\section{Overall observation about capable classifiers and statistical features}

It is challenging to suggest that a specific classifier should be capable for seizure detection. If we discuss classifiers, three constraints are very important whilst selecting a classifier-able to handle the high-dimensional dataset, high accuracy of the model, and able to retrieve the sensible knowledge. Not all machine learning classifiers are suitable for seizure detection and knowledge discovery tasks, mainly because of their black-box nature. This means that the logic rules/patterns are not visible and understandable to data scientists. In 'non-black-box' classifiers amongst decision trees [53] and decision forests [54], only decision forest algorithms are more capable, because the logic rules and knowledge discovered by a single decision tree are often limited and insufficient. For example, if we build a decision tree on a training dataset-it provides a limited or single set of logic rules and stops growing the tree further as all the data points in the training set accept that rule. On the other hand, if we build a decision forest on the same training set, we get multiple decision trees with more sensible logic rules. Siddiqui et al. [104] have done the analysis on CHB-MIT dataset to know which classifier performs better. For this, they applied two black-box (SVM and KNN) and two non-black-box (decision tree and ensemble of trees i.e., bagging, random subspace, boosting); they found non-black box classifier (ensemble) outperforms compared to other classifiers of black-box. Even ensemble also performs better than a single decision tree which is a non-black box classifier. Siddiqui et al. [63] applied two decision forests-Systematic Forest (SysFor) and Forest CERN for quick seizure detection using the concept of epoch length reduction. They achieved 100\% of accuracy. Similarly, Hussein et al. [100] also achieved $100 \%$ accuracy using decision forest-random forest approach.

The literature reveals that in the last few years, 'nonblack-box' classifiers, particularly decision forest approach, were widely used on brain datasets of EEG and ECoG for different research goals [76, 82, 94, 144]. The reasons for using the decision forest for seizure detection are as follows:

1. A decision forest overcomes some of the disadvantages of a decision tree. A decision tree discovers only a single set of logic rules from an input dataset. The logic rules that are discovered by a single decision tree may fail to correctly predict and classify the class values;

2. A decision forest can produce more set of logic rules/ patterns compared to a single decision tree and there is a high chance of good prediction/classification compared to a single decision tree;

3. Able to handle high-dimensional sets;

4. Due to its ensemble nature a decision forest mostly produces a high accuracy compared to a single tree and other classifiers [54];

5. Less computational time (specifically for Random forest);

6. Logic rules are clear and humanly interpretable such as analysts/domain experts can easily understand and suggest best opinions. For example, affected brain lobe by seizure, identifying suitable statistical features, etc.

Furthermore, many statistical features have been used for seizure detection. However, a comparison between them 
is difficult because of their heterogeneous nature. Some researchers used a single feature such as energy and entropy. On the other hand, a combination of statistical features such as energy, kurtosis, line length, entropy, skewness, max, standard deviation, and min may produce promising outcomes. Most research [34, 46, 92, 100, $109,145]$ have achieved better results using these features. The novelty of $[29,63,104,125]$ is the selected nine statistical features are able to assist in seizure detection with high accuracy, i.e., $100 \%$. This also provides the clue about seizure localization with the help of sensible logical rules. Hence, the selected group of features will not be a burden to the machine learning classifier but it will assist in related knowledge discovery.

\section{Research directions in seizure detection}

In this research analysis, we surveyed different machine learning classifiers used for seizure detection. No doubt, the progress of the persistent attempt has been found in this topic but few interesting research questions are also raised. In this section, we identify significant challenges which can uplift the future research in this area.

1. Selecting suitable statistical features and machine learning classifiers to take less computation time as the dataset has a high volume with a high dimension.

2. Accurate seizure detection on imbalanced datasets of long duration EEG recording datasets.

3. Quick seizure detection on long-hour EEG recording.

4. Whilst selecting the machine classifier it should be kept in mind that the classifier does not miss any necessary EEG channel/electrode.

5. Knowledge discovery from machine learning classifiers such as seizure localization which exactly points affected brain lobe(s), channel importance, and based on participating channels in seizure a knowledge could be provided to neurologist or neurosurgeon for suggesting epilepsy category.

\section{Conclusion}

With the increase of epilepsy, its accurate detection becomes increasingly important. A major challenge is to detect seizures correctly from a large volume of data. Due to the complexity of EEG signals in such datasets, machine learning classifiers are suitable for accurate seizure detection. Selecting suitable classifiers and features are, however, crucial.

As such, this paper has comprehensively reviewed machine learning approaches for seizure detection. As a result, we conclude that 'non-black-box' classifiers-decision forest (ensemble of decision trees) is most effective. This is because it can produce multiple sensible, explanatory logic rules with high accuracy of prediction. Further, it can help discover some relevant information such as seizure localization and exploring seizure types. On the contrary, 'black-box' classifiers cannot generate logic rules, although they can achieve high predictive accuracy. As for selecting suitable features, we should select those that can provide logical results. By the review of the literature, the use of the features such as entropy, line length, energy, skewness, kurtosis, and standard deviation can achieve $100 \%$ accuracy in the classifiers. We suggest not to use the irrelevant features as the dimension of the data increases. This is because the computation cost of a classifier will grow high, and it may also produce insensible patterns. If we use just one or two features such as line length and energy, the low-dimensional dataset will be generated. However, this dataset will not be fruitful for the knowledge discovery process.

This review paper has provided new perspectives to data scientists who are working on epileptic seizure detection using EEG signals. In summary, this paper focuses on the review of selecting machine learning classifiers and suitable features.

\section{Acknowledgements \\ We acknowledge the contribution of Dr. Khudeja Khatoon, MD, Faculty member of the Hayat Unani Medical College \& Research Centre, India for carefully looking the medical terminologies in the paper. Also, thankful to Mr. Mohammad Arshad, English language expert, Shoumou Investment and Trad- ing Company, KSA for proofreading the paper.}

\section{Authors' contributions}

MKS: acquisition of related works, machine learning study, comparative study, arguments, writeup. RMM reviewed the overall paper and provided com-

ments. $\mathrm{XH}$ : review the article on Machine Learning perspective, main contributions and direction of research. $\mathrm{NH}$ : discussion and table analysis. All authors read and approved the final manuscript.

Funding

Not applicable.

Availability of data and materials

Not applicable.

Competing interests

The authors declare that they have no competing interests.

Author details

${ }^{1}$ School of Engineering and Sciences, Tecnologico de Monterrey, Av. E. Garza Sada 2501, Monterrey, Nuevo Leon, Mexico. ${ }^{2}$ School of Computing and Mathematics, Charles Sturt University, 2640 Albury, NSW, Australia. ${ }^{3}$ College of Applied Studies and Community Service, King Saud University, Riyadh, Kingdom of Saudi Arabia.

Received: 24 December 2019 Accepted: 9 May 2020

Published online: 25 May 2020 


\section{References}

1. Organization WH (2006) Neurological disorders: public health challenges. World Health Organization, New York

2. Chaudhary UJ, Duncan JS, Lemieux L (2011) A dialogue with historical concepts of epilepsy from the babylonians to hughlings jackson: persistent beliefs. Epilepsy Behav 21(2):109-114

3. Reynolds EH (2009) Milestones in epilepsy*. Epilepsia 50(3):338-342. https://doi.org/10.1111/j.1528-1167.2009.02050.x

4. WHO (2005) Atlas: Epilepsy care in the world. World Health Organization, Geneva

5. Alarcón G, Valentín A (2012) Introduction to Epilepsy. Cambridge University Press, Cambridge

6. Qu H, Gotman J (1993) Improvement in seizure detection performance by automatic adaptation to the eeg of each patient. Electroencephalogr Clin Neurophysiol 86(2):79-87

7. Sazgar M, Young MG (2019) Seizures and Epilepsy. In: Absolute Epilepsy and EEG rotation review, pp. 9-46. Springer, New York

8. Schachter S, Shafer P, Sirven J (2013) What causes epilepsy and seizures. Epilepsy Foundation

9. Delanty N, Vaughan CJ, French JA (1998) Medical causes of seizures. Lancet 352(9125):383-390

10. WHO: Media Center Epilepsy, (Fact sheet N999). http://www.who.int/ mediacentre/factsheets/fs999/en/ (2015) Accessed 15 July 2019

11. Shafer PO, Sirven JI (2014) Epilepsy statistics. Epilepsy Foundation

12. Hannah JA, Brodie MJ (1998) Epilepsy and learning disabilities-a challenge for the next millennium? Seizure 7(1):3-13

13. Thurman DJ, Beghi E, Begley CE, Berg AT, Buchhalter JR, Ding D, Hesdorffer DC, Hauser WA, Kazis L, Kobau R et al (2011) Standards for epidemiologic studies and surveillance of epilepsy. Epilepsia 52(s7):2-26

14. Fisher RS (2017) The new classification of seizures by the international league against epilepsy 2017. Curr Neurol Neurosci Rep 17(6):48

15. Mahmud M, Kaiser MS, Hussain A, Vassanelli S (2018) Applications of deep learning and reinforcement learning to biological data. IEEE Trans Neural Networks Learn Syst 29(6):2063-2079

16. Mahmud M, Kaiser MS, Hussain A (2020) Deep learning in mining biological data. arXiv:2003.00108

17. Fayyad UM, Piatetsky-Shapiro G, Smyth P, Uthurusamy R (eds.) (1996) Advances in Knowledge Discovery and Data Mining. American Association for Artificial Intelligence, Menlo Park, CA, USA

18. Yin Y, Kaku I, Tang J, Zhu J (2011) Data mining: concepts, methods and applications in management and engineering design. Springer, New York

19. Islam MZ, D'Alessandro S, Furner M, Johnson L, Gray D, Carter L (2016) Brand switching pattern discovery by data mining techniques for the telecommunication industry in australia. Aust J Inform Syst. https://doi. org/10.3127/ajis.v20i0.1420

20. Aljumah AA, Ahamad MG, Siddiqui MK (2013) Application of data mining: diabetes health care in young and old patients. J King Saud Univ Comput Inform Sci 25(2):127-136

21. Aljumah A, Siddiqui M (2016) Data mining perspective: prognosis of life style on hypertension and diabetes. Int Arab J Inf Technol 13(1)

22. Siddiqui MK, Morales-Menendez R, Gupta PK, lqbal HM, Hussain F, Khatoon K, Ahmad S (2020) Correlation between temperature and COVID-19 (suspected, confirmed and death) cases based on machine learning analysis. J Pure Appl Microbiol 14(Spl Edn.)

23. Aljumah AA, Siddiqui MK (2014) Hypertension interventions using classification based data mining. Res J Appl Sci Eng Technol 7(17):3593-3602

24. Almazyad AS, Ahamad MG, Siddiqui MK, Almazyad AS (2010) Effective hypertensive treatment using data mining in Saudi Arabia. J Clin Monit Comput 24(6):391-401

25. Singh GA, Gupta PK (2019) Performance analysis of various machine learning-based approaches for detection and classification of lung cancer in humans. Neural Comput Appl 31(10):6863-6877

26. Fu T-c (2011) A review on time series data mining. Eng Appl Artif Intell 24(1):164-181

27. Tzallas AT, Tsipouras MG, Tsalikakis DG, Karvounis EC, Astrakas L, Konitsiotis S, Tzaphlidou M (2012) Automated epileptic seizure detection methods: a review study. In: Epilepsy-histological, electroencephalographic and psychological aspects. InTech

28. Abbasi B, Goldenholz DM (2019) Machine learning applications in epilepsy. Epilepsia

29. Siddiqui MK. Brain data mining for epileptic seizure-detection. Doctoral Dissertation, Charles Sturt University, Australia

30. Paul Y (2018) Various epileptic seizure detection techniques using biomedical signals: a review. Brain Inform 5(2):6

31. Boonyakitanont P, Lek-uthai A, Chomtho K, Songsiri J (2020) A review of feature extraction and performance evaluation in epileptic seizure detection using eeg. Biomed Signal Process Control 57:101702

32. Sharmila A, Geethanjali P (2019) A review on the pattern detection methods for epilepsy seizure detection from EEG signals. Biomed Eng / Biomedizinische Technik. 64(5):507-17

33. van Westrhenen A, De Cooman T, Lazeron RH, Van Huffel S, Thijs RD (2019) Ictal autonomic changes as a tool for seizure detection: a systematic review. Clin Auton Res 29(2):161-181

34. Logesparan AJC Lojini, Rodriguez-Villegas E (2012) Optimal features for online seizure detection. Med Biol Eng Comput 50(7):659-669

35. Ramgopal S, Thome-Souza S, Jackson M, Kadish NE, Fernández IS, Klehm J, BosI W, Reinsberger C, Schachter S, Loddenkemper T (2014) Seizure detection, seizure prediction, and closed-loop warning systems in epilepsy. Epilepsy Behav 37:291-307

36. Quintero-Rincón A, D'Giano C, Batatia H (2019) Seizure onset detection in eeg signals based on entropy from generalized gaussian pdf modeling and ensemble bagging classifier. In: Digital Health Approach for Predictive, Preventive, Personalised and Participatory Medicine, pp. 1-10. Springer, New York

37. Greene B, Faul S, Marnane W, Lightbody G, Korotchikova I, Boylan G (2008) A comparison of quantitative eeg features for neonatal seizure detection. Clin Neurophysiol 119(6):1248-1261

38. Gotman J, Ives J, Gloor P (1979) Automatic recognition of inter-ictal epileptic activity in prolonged eeg recordings. Electroencephalogr Clin Neurophysiol 46(5):510-520

39. Gotman J (1982) Automatic recognition of epileptic seizures in the eeg. Electroencephalogr Clin Neurophysiol 54(5):530-540

40. Koffler D, Gotman J (1985) Automatic detection of spike-and-wave bursts in ambulatory eeg recordings. Electroencephalogr Clin Neurophysiol 61(2):165-180

41. Shoeb A, Guttag J (2010) Application of machine learning to epileptic seizure detection. In: 2010 the 27th International Conference on Machinelearning, Haifa, Israel

42. Dorai A, Ponnambalam K (2010) Automated epileptic seizure onset detection. In: Autonomous and Intelligent Systems (AIS), 2010 International Conference On, pp. 1-4 . IEEE

43. Lahmiri S (2018) An accurate system to distinguish between normal and abnormal electroencephalogram records with epileptic seizure free intervals. Biomed Sign Process Control 40:312-317

44. Donos C, Dümpelmann M, Schulze-Bonhage A (2015) Early seizure detection algorithm based on intracranial EEG and random forest classification. Int J Neur Syst 25(05):1550023

45. Ocak H (2009) Automatic detection of epileptic seizures in eeg using discrete wavelet transform and approximate entropy. Exp Syst Appl 36(2):2027-2036

46. Thomas E, Temko A, Lightbody G, Marnane W, Boylan G (2011) Advances in automated neonatal seizure detection. New Adv Intell Sign Process 1:93-113

47. Al Ghayab HR, Li Y, Abdulla S, Diykh M, Wan X (2016) Classification of epileptic EEG signals based on simple random sampling and sequential feature selection. Brain Inform 3(2):85-91

48. Herwig U, Satrapi P, Schönfeldt-Lecuona C (2003) Using the international 10-20 eeg system for positioning of transcranial magnetic stimulation. Brain Topogr 16(2):95-99

49. Misulis KE (2013) Atlas of EEG, seizure semiology, and management Oxford University Press, Oxford

50. Sammut C, Webb Gl (2017) Encyclopedia of machine learning and data mining. Springer, New York

51. Adnan MN, Islam MZ (2016) Forest CERN: A new decision forest building technique. In: Pacific-Asia Conference on Knowledge Discovery and Data Mining, pp. 304-315. Springer, New York 
52. Cortes C, Vapnik V (1995) Support-vector networks. Mach Learn 20(3):273-297

53. Quinlan JR (1993) C4.5: Programs for machine learning. Morgan Kaufmann Publishers Inc., San Francisco

54. Breiman L (2001) Random forests. Mach Learn 45(1):5-32. https://doi. org/10.1023/A:1010933404324

55. Arlot S, Celisse A et al (2010) A survey of cross-validation procedures for model selection. Statistics surveys 4:40-79

56. Kurgan LA, Cios KJ (2004) CAIM discretization algorithm. IEEE Trans Knowl Data Eng 16(2):145-153

57. Li J, Liu H (2003) Ensembles of cascading trees. In: Data Mining, 2003. ICDM 2003. Third IEEE International Conference On, pp. 585-588. IEEE

58. Powers DM (2011) Evaluation: from precision, recall and f-measure to roc, informedness, markedness and correlation

59. Goldberger A, Amaral L, Glass L, Hausdorff J, Ivanov PC, Mark R, Mietus J, Moody G, Peng C, Stanley H (2000) PhysioBank, PhysioToolkit, and PhysioNet: components of a new research resource for complex physiologic signals. Circulation 101(23):e215-e220

60. CHB-MIT Scalp EEG Database. https://physionet.org/pn6/chbmit/. Accessed 20 Jun 2015

61. Shoeb A, Edwards H, Connolly J, Bourgeois B, Treves ST, Guttag J (2004) Patient-specific seizure onset detection. Epilepsy Behav 5(4):483-498

62. Kramer MA, Kolaczyk ED, Kirsch HE (2008) Emergent network topology at seizure onset in humans. Epilepsy Res 79(2):173-186

63. Siddiqui MK, Islam MZ, Kabir MA (2018) A novel quick seizure detection and localization through brain data mining on ecog dataset. Neural Computing and Applications 1-14

64. Freiburg seizure prediction project. Freiburg, Germany. http://epile psy.uni-freiburg.de/freiburg-seizure-prediction-project/eeg-database (2003)

65. Andrzejak RG, Lehnertz K, Mormann F, Rieke C, David P, Elger CE (2001) Indications of nonlinear deterministic and finite-dimensional structures in time series of brain electrical activity: Dependence on recording region and brain state. Phys Rev E 64(6):061907

66. Andrzejak RG, Schindler K, Rummel C (2012) Nonrandomness, nonlinear dependence, and nonstationarity of electroencephalographic recordings from epilepsy patients. Phys Rev E 86(4):046206

67. Lee H, Kim S (2016) Black-box classifier interpretation using decision tree and fuzzy logic-based classifier implementation. Int J Fuzzy Logic Intell Syst 16(1):27-35

68. Adnan MN, Islam MZ (2017) Forex++: A new framework for knowledge discovery from decision forests. Aust J Inform Syst. https://doi. org/10.3127/ajis.v21i0.1539

69. Guo L, Rivero D, Dorado J, Rabunal JR, Pazos A (2010) Automatic epileptic seizure detection in eegs based on line length feature and artificial neural networks. J Neurosci Methods 191(1):101-109

70. Koolen N, Jansen K, Vervisch J, Matic V, De Vos M, Naulaers G, Van Huffel S (2014) Line length as a robust method to detect high-activity events: automated burst detection in premature eeg recordings. Clin Neurophysiol 125(10):1985-1994

71. Logesparan L, Rodriguez-Villegas E, Casson AJ (2015) The impact of signal normalization on seizure detection using line length features Med Biol Eng Comput 53(10):929-942

72. Song Y, Liò P (2010) A new approach for epileptic seizure detection: sample entropy based feature extraction and extreme learning machine. J Biomed Sci Eng 3(06):556

73. Zhang $Y$, Zhang $Y$, Wang J, Zheng $X$ (2014) Comparison of classification methods on EEG signals based on wavelet packet decomposition. Neural Comput Appl 26(5):1217-1225. https://doi.org/10.1007/s0052 1-014-1786-7

74. Ahmad MA, Khan NA, Majeed W (2014) Computer assisted analysis system of electroencephalogram for diagnosing epilepsy. In: Pattern Recognition (ICPR), 2014 22nd International Conference On, pp. 3386-3391. IEEE

75. Gill AF, Fatima SA, Akram MU, Khawaja SG, Awan SE (2015) Analysis of eeg signals for detection of epileptic seizure using hybrid feature set. In: Theory and Applications of Applied Electromagnetics, pp. 49-57. Springer

76. Orellana MP, Cerqueira F (2016) Personalized epilepsy seizure detection using random forest classification over one-dimension transformed EEG data. bioRxiv, 070300
77. Birjandtalab J, Pouyan MB, Cogan D, Nourani M, Harvey J (2017) Automated seizure detection using limited-channel eeg and non-linear dimension reduction. Comput Biol Med 82:49-58

78. Yan A, Zhou W, Yuan Q, Yuan S, Wu Q, Zhao X, Wang J (2015) Automatic seizure detection using stockwell transform and boosting algorithm for long-term eeg. Epilepsy Behav 45:8-14

79. Amin HU, Malik AS, Ahmad RF, Badruddin N, Kamel N, Hussain M, Choo W-T (2015) Feature extraction and classification for eeg signals using wavelet transform and machine learning techniques. Austr Phys Eng Sci Med 38(1):139-149

80. Mursalin M, Zhang Y, Chen Y, Chawla NV (2017) Automated epileptic seizure detection using improved correlation-based feature selection with random forest classifier. Neurocomputing 241:204-214

81. Zabihi M, Kiranyaz S, Ince T, Gabbouj M (2013) Patient-specific epileptic seizure detection in long-term eeg recording in paediatric patients with intractable seizures

82. Truong ND, Kuhlmann L, Bonyadi MR, Yang J, Faulks A, Kavehei O (2017) Supervised learning in automatic channel selection for epileptic seizure detection. Exp Syst Appl

83. Fergus $\mathrm{P}$, Hussain A, Hignett D, Al-Jumeily D, Abdel-Aziz K, Hamdan $\mathrm{H}$ (2016) A machine learning system for automated whole-brain seizure detection. Appl Comput Inform 12(1):70-89

84. Chen D, Wan S, Xiang J, Bao FS (2017) A high-performance seizure detection algorithm based on discrete wavelet transform (dwt) and eeg. PLOS ONE 12(3):0173138

85. Satapathy SK, Jagadev AK, Dehuri S (2017) Weighted majority voting based ensemble of classifiers using different machine learning techniques for classification of eeg signal to detect epileptic seizure. Informatica 41(1):99

86. Yuan Q, Zhou W, Zhang L, Zhang F, Xu F, Leng Y, Wei D, Chen M (2017) Epileptic seizure detection based on imbalanced classification and wavelet packet transform. Seizure

87. Subasi A, Kevric J, Canbaz MA (2019) Epileptic seizure detection using hybrid machine learning methods. Neural Comput Appl 31(1):317-325

88. Al Ghayab HR, Li Y, Siuly S, Abdulla S (2019) Epileptic seizures detection in eegs blending frequency domain with information gain technique. Soft Comput 23(1):227-239

89. Selvakumari RS, Mahalakshmi M, Prashalee P (2019) Patient-specific seizure detection method using hybrid classifier with optimized electrodes. J Med Syst 43(5):121

90. Chen S, Zhang X, Chen L, Yang Z (2019) Automatic diagnosis of epileptic seizure in electroencephalography signals using nonlinear dynamics features. IEEE Access 7:61046-61056

91. Birjandtalab J, Jarmale VN, Nourani M, Harvey J (2018) Imbalance learning using neural networks for seizure detection. In: 2018 IEEE Biomedical Circuits and Systems Conference (BioCAS), pp. 1-4 . IEEE

92. Lahmiri S, Shmuel A (2018) Accurate classification of seizure and seizure-free intervals of intracranial eeg signals from epileptic patients. IEEE Trans Instrum Meas 68(3):791-796

93. Tzimourta KD, Tzallas AT, Giannakeas N, Astrakas LG, Tsalikakis DG, Angelidis P, Tsipouras MG (2019) A robust methodology for classification of epileptic seizures in eeg signals. Health Technol 9(2):135-142

94. Wang X, Gong G, Li N, Qiu S (2019) Detection analysis of epileptic EEG using a novel random forest model combined with grid search optimization. Front Human Neurosci 13:12

95. Raghu S, Sriraam N (2018) Classification of focal and non-focal eeg signals using neighborhood component analysis and machine learning algorithms. Exp Syst Appl 113:18-32

96. Alickovic E, Kevric J, Subasi A (2018) Performance evaluation of empirical mode decomposition, discrete wavelet transform, and wavelet packed decomposition for automated epileptic seizure detection and prediction. Biomed Sign Process Contr 39:94-102

97. Fasil O, Rajesh R (2019) Time-domain exponential energy for epileptic eeg signal classification. Neurosci Lett 694:1-8

98. Manzouri F, Heller S, Dümpelmann M, Woias P, Schulze-Bonhage A (2018) A comparison of machine learning classifiers for energyefficient implementation of seizure detection. Front Syst Neurosci 12: 43 
99. Parvez MZ, Paul M (2014) Epileptic seizure detection by analyzing eeg signals using different transformation techniques. Neurocomputing 145:190-200

100. Hussein R, Elgendi M, Wang ZJ, Ward RK (2018) Robust detection of epileptic seizures based on I1-penalized robust regression of EEG signals. Exp Syst Appl 104:153-167

101. Mursalin M, Islam SS, Noman MK (2019) Epileptic seizure classification using statistical sampling and a novel feature selection algorithm. arXiv preprint arXiv:1902.09962

102. Sharma RR, Varshney P, Pachori RB, Vishvakarma SK (2018) Automated system for epileptic EEG detection using iterative filtering. IEEE Sens Lett 2(4):1-4

103. Tzallas AT, Tsipouras MG (2007) Fotiadis DI (2007) Automatic seizure detection based on time-frequency analysis and artificial neural networks. Comput Intell Neurosci 2007:80510

104. Siddiqui MK, Islam MZ, Kabir MA (2017) Analyzing performance of classification techniques in detecting epileptic seizure. In: International Conference on Advanced Data Mining and Applications, pp. 386-398. Springer, New York

105. Sanei S, Chambers JA (2013) EEG signal processing. Wiley, New York

106. Chaovalitwongse WA, Prokopyev OA, Pardalos PM (2006) Electroencephalogram (eeg) time series classification: applications in epilepsy. Ann Ope Res 148(1):227-250

107. Moselhy HF (2011) Psychosocial and cultural aspects of epilepsy. In: Novel Aspects on Epilepsy. InTech

108. Esteller R, Echauz J, Tcheng T, Litt B, Pless B (2001) Line length: an efficient feature for seizure onset detection. In: Engineering in Medicine and Biology Society, 2001. Proceedings of the 23rd Annual International Conference of the IEEE, vol. 2, pp. 1707-1710. IEEE

109. Guerrero-Mosquera C, Trigueros AM, Franco Jl, Navia-Vázquez Á (2010) New feature extraction approach for epileptic eeg signal detection using time-frequency distributions. Med Biol Eng Comput 48(4):321-330

110. Olsen DE, Lesser RP, Harris JC, Webber WRS, Cristion JA (1994) Automatic detection of seizures using electroencephalographic signals. Google Patents. US Patent 5,311, 876

111. Acharya UR, Molinari F, Sree SV, Chattopadhyay S, Ng K-H, Suri JS (2012) Automated diagnosis of epileptic EEG using entropies. Biomed Sign Process Contr 7(4):401-408

112. Kannathal N, Choo ML, Acharya UR, Sadasivan P (2005) Entropies for detection of epilepsy in eeg. Comput Meth Progr Biomed 80(3):187-194

113. Kumar Y, Dewal M (2011) Complexity measures for normal and epileptic eeg signals using apen, sampen and sen. IJCCT 2(7):6-12

114. Shimizu M, liya M, Fujii H, Kimura S, Suzuki M, Nishizaki M (2019) Left ventricular end-systolic contractile entropy can predict cardiac prognosis in patients with complete left bundle branch block. Journal of Nuclear Cardiology 1-10

115. Pal PR, Panda R (2010) Classification of eeg signals for epileptic seizure evaluation. In: Students'Technology Symposium (TechSym), 2010 IEEE, pp. 72-76. IEEE

116. Cepukenas J, Lin C, Sleeman D (2015) Applying rule extraction and rule refinement techniques to (blackbox) classifiers. In: Proceedings of the 8th international conference on knowledge capture, p. 27. ACM

117. Birjandtalab J, Pouyan MB, Nourani M (2016) Unsupervised eeg analysis for automated epileptic seizure detection. In: Proceedings of the first international workshop on pattern recognition, international society for optics and photonics, pp. 100110-100110

118. Abualsaud K, Mahmuddin M, Saleh M, Mohamed A (2015) Ensemble classifier for epileptic seizure detection for imperfect eeg data. Sci World J 2015:945689

119. Chen C, Liu J, Syu J (2012) Application of chaos theory and data mining to seizure detection of epilepsy. Proc Conf. IPCSIT/Hong Kong 25:23-28

120. Polat K, Güneş S (2007) Classification of epileptiform eeg using a hybrid system based on decision tree classifier and fast fourier transform. Appl Math Comput 187(2):1017-1026

121. Quinlan R (2004) Data mining tools see5 and c5. 0

122. Cios KJ, Pedrycz W, Swiniarski RW, Kurgan LA (2007) Data mining: a knowledge discovery approach. Springer, New York

123. Islam MZ, Giggins H (2011) Knowledge discovery through sysfor: a systematically developed forest of multiple decision trees. In: Proceedings of the Ninth Australasian Data Mining Conference-Volume 121, pp. 195-204. Australian Computer Society, Inc

124. Ho TK (1998) The random subspace method for constructing decision forests. IEEE Trans Patt Analy Mach Intell 20(8):832-844

125. Siddiqui MK, Islam MZ (2016) Data mining approach in seizure detection. In: 2016 IEEE Region 10 Conference (TENCON), Singapore, pp. 3579-3583. Institute of Electrical and Electronics Engineers (IEEE), https ://doi.org/10.1109/tencon.2016.7848724

126. Hosseini M-P, Pompili D, Elisevich K, Soltanian-Zadeh H (2018) Random ensemble learning for eeg classification. Artif Intell Med 84:146-158

127. Hassan AR, Siuly S, Zhang Y (2016) Epileptic seizure detection in eeg signals using tunable-q factor wavelet transform and bootstrap aggregating. Comput Meth Prog Biomed 137:247-259

128. Hosseini M-P, Hajisami A, Pompili D (2016) Real-time epileptic seizure detection from eeg signals via random subspace ensemble learning. In: Autonomic Computing (ICAC), 2016 IEEE International Conference On, pp. 209-218. IEEE

129. Jurcak V, Tsuzuki D, Dan I (2007) 10/20, 10/10, and 10/5 systems revisited: Their validity as relative head-surface-based positioning systems. Neurolmage 34(4):1600-1611. https://doi.org/10.1016/j.neuroimage 2006.09 .024

130. Fakhraei S, Soltanian-Zadeh H, Fotouhi F, Elisevich K (2011) Confidence in medical decision making: Application in temporal lobe epilepsy data mining. In: Proceedings of the 2011 Workshop on Data Mining for Medicine and Healthcare. DMMH '11, pp. 60-63. ACM, New York, NY, USA . https://doi.org/10.1145/2023582.2023593

131. Keraudren K, Kainz B, Oktay O, Kyriakopoulou V, Rutherford M, Hajnal JV, Rueckert D (2015) Automated localization of fetal organs in mri using random forests with steerable features. In: International conference on medical image computing and computer-assisted Intervention, pp. 620-627. Springer, New York

132. Trans-Cranial-Technologies: 10/20 System Positioning. https://www. trans-cranial.com/local/manuals/10_20_pos_man_v1_0_pdf.pdf. Accessed 7 Oct 2015

133. Acar E, Bingöl CA, Bingöl H, Yener B (2006) Computational analysis of epileptic focus localization. In: Proceedings of the 24th IASTED International Conference on Biomedical Engineering. BioMed'06, pp. 317-322. ACTA Press, Anaheim, CA, USA. http://dl.acm.org/citation. cfm?id=1166506.1166562

134. Ghannad-Rezaie M, Soltanain-Zadeh H, Siadat MR, Elisevich KV (2006) Medical data mining using particle swarm optimization for temporal lobe epilepsy. In: 2006 IEEE International conference on evolutionary computation, pp. 761-768. https://doi.org/10.1109/CEC.2006.1688388

135. Mansouri A, Singh SP, Sayood K (2019) Online eeg seizure detection and localization. Algorithms 12(9):176

136. Rai K, Bajaj V, Kumar A (2015) Features extraction for classification of focal and non-focal eeg signals. Inform Sci Appl Lect Notes Electr Eng 339:599-605

137. Hadoush $\mathrm{H}$, Alafeef M, Abdulhay E (2019) Brain complexity in children with mild and severe autism spectrum disorders: Analysis of multiscale entropy in eeg. Brain topography 1-8

138. Fernández-Delgado M, Cernadas E, Barro S, Amorim D (2014) Do we need hundreds of classifiers to solve real world classification problems? J Mach Learn Res 15(1):3133-3181

139. Breiman L (1996) Bagging predictors. Mach Learn 24(2):123-140

140. Galar M, Fernandez A, Barrenechea E, Bustince H, Herrera F (2012) A review on ensembles for the class imbalance problem: bagging-, boosting-, and hybrid-based approaches. IEEE Trans Syst Man Cybern C 42(4):463-484

141. Yang F, Wang H-Z, Mi H, Cai W-W et al (2009) Using random forest for reliable classification and cost-sensitive learning for medical diagnosis. BMC Bioinform 10(1):22

142. El Saadi H, Al-Sadek AF, Fakhr MW (2012) Informed under-sampling for enhancing patient specific epileptic seizure detection. Int J Comput Appl 57:16

143. Amin S, Kamboh AM (2016) A robust approach towards epileptic seizure detection. In: Machine Learning for Signal Processing (MLSP), 2016 IEEE 26th International Workshop On, pp. 1-6. IEEE

144. Yang Z, Choupan J, Reutens D, Hocking J (2015) Lateralization of temporal lobe epilepsy based on resting-state functional magnetic resonance imaging and machine learning. Front Neurol 6:184 
145. Tito M, Cabrerizo M, Ayala M, Jayakar P, Adjouadi M (2009) Seizure detection: an assessment of time-and frequency-based features in a unified two-dimensional decisional space using nonlinear decision functions. J Clin Neurophysiol 26(6):381-391

\section{Publisher's Note}

Springer Nature remains neutral with regard to jurisdictional claims in published maps and institutional affiliations.
Submit your manuscript to a SpringerOpen ${ }^{\odot}$ journal and benefit from:

- Convenient online submission

- Rigorous peer review

- Open access: articles freely available online

- High visibility within the field

- Retaining the copyright to your article

Submit your next manuscript at $\boldsymbol{\nabla}$ springeropen.com 